\title{
Moroccan Taxi Drivers Fatigue Using Pichot Questionnaire: A Cross-Sectional Survey
}

Nadia Benaicha, Abdessalam Diarra, Oussmane Désiré Diakite, Oussamne S.Y., Adil Najdi, Mohamed Amine Berraho

1 Department of Epidemiology and Community Health, Faculty of Medicine, University Sidi Mohamed Benabdellah, Fez, Morocco

2 Department of Epidemiology and Community Health, Faculty of Medicine, University Abdelmalek Essaadi, Tangier, Morocco

\begin{abstract}
Studies conducted in many countries the world has shown that fatigued driving contributes to approximately 3\%-30\% of road accidents. We conducted this study to estimate the prevalence of fatigue among taxi drivers and to assess the determinant of fatigue among taxi drivers. In this cross-sectional study, 300 urban and rural taxi drivers from Fez, Morocco were enrolled from January to May 2013. The mean score of Pichot scale was $13.59 \pm 8.23$. The mean duration of sleep was $6.78 \pm 1.47 \mathrm{~h}$ per day. The mean rate of accident was $2.78 \pm 1.12$ per year. At the final model of the multiple linear regression we found an association statistically significant between Pichot scale and income, cardiovascular disorders, tobacco use, problems with colleagues, regularity of sleep and sleeping at the wheel. This study opened the door to many discussions about the professional drivers' conditions of work and we can argue that we should ameliorate the professional drivers conditions of work in Morocco.
\end{abstract}

Key words: Fatigue, Pichot scale, taxi drivers, Morocco.

\section{Introduction}

Fatigue is a state of tiredness which can perturb a person attention and ability to take good decision [1-4]. And hence driving when fatigued can make the driver falling asleep and as consequence the risk of accident may be very high. We can easily understand that driving when fatigued causes thousands of road traffic accidents and fatalities each year. Many international studies found that fatigue can be a probable major cause of truck driver accidents (about $20 \%$ to more than $50 \%$ ) [5-7]. Studies conducted in many countries the world has shown that fatigued driving contributes to approximately $3 \%-30 \%$ of road accidents [12].

The World Health Organization estimates that by 2020 road traffic injuries will represent the third major cause of disability adjusted years of life lost worldwide [13]. In Morocco, according to statistics of the Ministry of equipment, transport and logistics, the

Corresponding author: Nadia Benaicha, Ph.D., research fields: health care management. number of accidents has reached 35.769 between January and June 2015. The official statistics reported 4.364 serious injuries and 46.401 slightly injured [13]. And the sector of transport and especially taxi transport is very informal and there are no official guidelines on the work hours and rest periods of taxi drivers. Since fatigued driving continues to threaten thousands of citizens and professionals lives.

Despite the importance of quality of sleep in drivers, there were few researches concerning this topic in Morocco. We conducted this study to estimate the prevalence of fatigue among taxi drivers and to assess the determinant of fatigue among taxi drivers.

\section{Materiel and Methods}

In this cross-sectional study, 300 urban and rural taxi drivers from Fez, Morocco were enrolled from January to May 2013. All interviews were performed by two trained investigators. First all items on the questionnaire were explained to and discussed with the participants, and their questions were answered as they filled out the forms. 
The questionnaire includes socio-demographic data, medical problems, such as diabetes, cardiovascular problems or pulmonary disorders, addiction including tobacco, water pipe, alcohol or drug, sleep habits and different kind of stress. All drivers under study also completed forms to evaluate their fatigue using Pichot questionnaire [11]. Pathological tiredness is referred to when people find it harder to carry out their daily activities than in their usual condition. Pichot's subjective scale has been proposed to assess the importance of this handicap. Pichot's scale consists of eight questions and yields a score of 0 to 32 . It is easy to administer and is currently one of the most the most widely used for tiredeness.

All variables were summarized by using descriptive statistics. Categorical variables were described in terms of proportions and quantitative variables were described in terms of average extreme values and standard deviation.

The association between dependent variable Pichot's scale and several potential explanatory variables was investigated. Bi-variate analysis was used and the association between the quantitative variable (Pichot's scale) and many qualitative variables (sex, tobacco use...) was searched by $t$-test and was selected as the significance level a $P$ value of 0.05 . The association between the dependent variable (Pichot's scale) and quantitative variables was searched by simple linear regression and was selected as the significance level a $P$ value of 0.05 .

Multivariate analysis was made (multiple linear regression), the dependent variable was Pichot's scale and we include in the model all the explanatory variables which have a significance level at the bivariate analysis less or equal 0.20

Statistical analysis was done on SPSS Version 17.

\section{Results}

All participants were male and their mean age was $42.51 \pm 10.81$ (range 18-74) years. In terms of marital status, $45(15.0 \%)$ were single, 255 (84.7\%) were married, and $1(0.3 \%)$ was divorced and no one widowed. The mean BMI of the drivers was $26.81 \pm$ $4.31 \mathrm{~kg} / \mathrm{m}^{2}$. The self-report of associated diseases indicated heart disease in $4(1.3 \%)$, COPD in $1(0.3 \%)$, diabetes in $18(6.0 \%)$, other disease in $17(15.6 \%)$. In their history of drug abuse 97 (32.1\%) used tobacco, 15 (5.0\%) had alcohol abuse with mean duration of alcohol abuse $1.4 \pm 4.1$ (range $0-31$ ) and 25 (8.3\%) had a history of drug abuse. Our study population reported coffee and tea consumption respectively 230 (76.2\%) and $224(74.2 \%)$. Finally, $100(33.2 \%)$ drivers had regular physical activity.

Their experience in driving was $8.34 \pm 3.56$ years on average.7 $(2.3 \%)$ drivers were used to work by night, $172(57.1 \%)$ were used to work during the day and 122 (40.5\%) worked both during the day and by night Table1.

The mean score of Pichot scale was $13.59 \pm 8.23$ (range from 0 to 32) Figure 1. The mean duration of sleep was $6.78 \pm 1.47 \mathrm{~h}$ per day. The mean rate of accident was $2.78 \pm 1.12$ per year. We found a the simple linear regression significant association with Pichot scale and age $(P \leq 0.001)$, marital status with not married Vs married $(P=0.004)$, income with medium or high Vs low $(P=0.008)$, diabeteswith diabetes patients Vs subjects without diabetes $(P=$ 0.008), cardiovascular disorders with cardiovascular disorder patients Vs subjects without cardiovascular disorders $(P \leq 0.001)$, respiratory disorders with patients with respiratory disorders Vs patients without respiratory disorders $(P \leq 0.000)$, tobacco use with subject who didn't use tobacco Vs subjects who used tobacco $(P=0.035)$, alcohol use with subject who used alcohol Vs subject who didn't use alcohol $(P=0.014)$, coffee consumption with subject who used coffee Vs subject who didn't use coffee $(P=0.012)$, problems with colleagues with subjects who didn't have problems with colleagues Vs subject who had problems with colleagues $(P \leq 0.000)$, problems with clients with subjects who didn't have problems with clients Vs subject who had problems with clients $(P=$ 
0.025), regularity of sleep wit subject who didn't have regular sleeping Vs subject who had regular sleeping $(P=0.002)$, sleeping at the wheel with subject who didn't sleep at the wheel Vs subject who slept at the wheel $(P=0.002)$, road accidents with subject who never had accident last year Vs subject who had at least one accident last year $(P \leq 0.000)$, deflection of the car with subject who never had car deflection with subject who had car deflection before $(P=0.001)$ Table 2.

Table 1 Descriptive data of the taxi drivers.

\begin{tabular}{lll}
\hline & Fequency $(\%)$ & Mean \pm SD \\
\hline Age in years $(\mathrm{n}=300)$ & & $48.2 \pm 10.8$ \\
Driving experience in years $(\mathrm{n}=300)$ & & $8.3 \pm 3.5$ \\
Working time $(\mathrm{n}=300)$ & $172(57.1 \%)$ & \\
Daily & $7(2.3 \%)$ & \\
By night & $122(40.5 \%)$ & \\
Both & $45(15.0 \%)$ & \\
Marital status $(\mathrm{n}=300)$ & $255(84.7 \%)$ & \\
Single & $1(0.3 \%)$ & \\
Married & $0(0.0 \%)$ & \\
Divorced & & \\
Widowed & $4(1.4 \%)$ & \\
BMI $($ Kg/m2) $(\mathrm{n}=294)$ & $1(0.4 \%)$ & \\
Heart disease $(\mathrm{n}=300)$ & $18(6.0 \%)$ & \\
COPD $(\mathrm{n}=250)$ & $17(15.6 \%)$ & \\
Diabetes $(\mathrm{n}=300)$ & $97(32.2 \%)$ & \\
Other diseases $(\mathrm{n}=109)$ & $15(5.0 \%)$ & \\
Tobacco use $(\mathrm{n}=300)$ & $25(8.3 \%)$ & \\
Alcohol abuse $(\mathrm{n}=300)$ & $230(76.2 \%)$ & \\
Drug abuse $(\mathrm{n}=300)$ & $224(74.2 \%)$ & \\
Coffee consumption $(\mathrm{n}=300)$ & $100(33.2 \%)$ & \\
Tea consumption $(\mathrm{n}=300)$ & & \\
Regular physical activity $(\mathrm{n}=300)$ & & \\
\hline
\end{tabular}

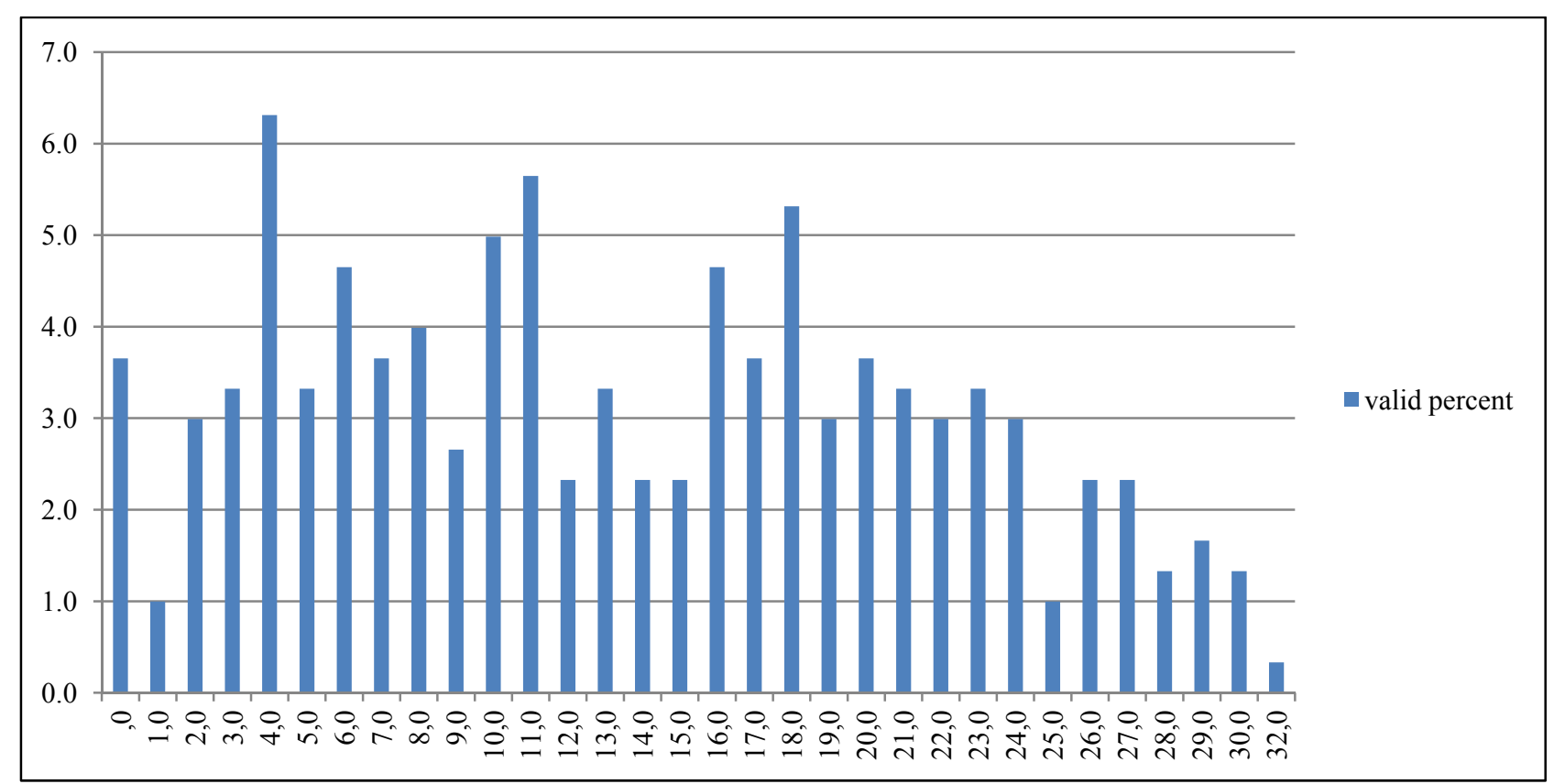

Fig. 1 Distribution of participants according to the Pichot scale. 
Table 2 Pichot scale and associated factors (Simple Linear Regression).

\begin{tabular}{|c|c|c|c|}
\hline & $\mathrm{B}$ & IC $(95 \%)$ & $\mathrm{p}$ \\
\hline Age & 0.226 & {$[0.008 ; 2.570]$} & 0.000 \\
\hline \multicolumn{4}{|l|}{ Marital status } \\
\hline Not married & 0.165 & {$[1.203 ; 6.294]$} & \multirow[t]{3}{*}{0.004} \\
\hline Married & 1 & - & \\
\hline Income & 1 & - & \\
\hline \multicolumn{4}{|l|}{ Low income } \\
\hline Diabetes & -0.158 & {$[-0.003 ; 0.000]$} & 0.008 \\
\hline Yes & 0.152 & {$[0.801 ; 5.374]$} & \multirow{2}{*}{0.008} \\
\hline No & 1 & - & \\
\hline \multicolumn{4}{|l|}{ Cardiovascular disorders } \\
\hline Yes & 0.300 & {$[4.330 ; 9.247]$} & \\
\hline No & 1 & - & 0.000 \\
\hline \multicolumn{4}{|l|}{ Respiratory disorders } \\
\hline Yes & 0.279 & & \\
\hline No & 1 & {$[4.484 ; 10.274]$} & 0.000 \\
\hline \multicolumn{4}{|l|}{ Tobacco use } \\
\hline Yes & 1 & - & \\
\hline No & -0.122 & {$[-3.084 ;-0.111]$} & 0.035 \\
\hline \multicolumn{4}{|l|}{ Alcohol use } \\
\hline Yes & 0.142 & {$[0.553 ; 4.872]$} & \multirow{2}{*}{0.014} \\
\hline No & 1 & - & \\
\hline \multicolumn{4}{|l|}{ Coffee consumption } \\
\hline Yes & -0.145 & {$[-5.023 ;-0.615]$} & \\
\hline No & 1 & - & 0.012 \\
\hline \multicolumn{4}{|l|}{ Problems with colleagues } \\
\hline Yes & 1 & - & \\
\hline No & $\begin{array}{l}1 \\
-0.219\end{array}$ & $\overline{-}-6.132 ;-2.007]$ & 0.000 \\
\hline \multicolumn{4}{|l|}{ Problems with clients } \\
\hline Yes & 1 & - & \\
\hline No & -0.129 & {$[-4.086 ;-0.272]$} & 0.025 \\
\hline \multicolumn{4}{|l|}{ Regularity of sleep } \\
\hline Yes & 1 & & \\
\hline No & 0.181 & {$[1.142 ; 4.825]$} & 0.002 \\
\hline \multicolumn{4}{|l|}{ Sleeping at the wheel } \\
\hline Yes & 1 & - & \\
\hline No & -0.200 & {$[-5.426 ;-1.235]$} & 0.002 \\
\hline \multicolumn{4}{|l|}{ Road accident last year } \\
\hline Yes & 1 & - & \\
\hline No & -0.230 & {$[-5.614 ;-1.966]$} & 0.000 \\
\hline \multicolumn{4}{|l|}{ Deflection of the car } \\
\hline Yes & 1 & - & \\
\hline No & -0.185 & {$[-5.020 ;-1.234]$} & 0.001 \\
\hline
\end{tabular}

At the final model of the multiple linear regression we found an association statistically significant between Pichot scale and income $(P=0.017)$, cardiovascular disorders $(P \leq 0.001)$, tobacco use $(P=0.010)$, problems with colleagues $(P=0.002)$, regularity of sleep $(P=0.013)$ and sleeping at the wheel $(P=0.002)$ Table 3.

\section{Discussion}

In this cross-sectional study, 300 urban and rural taxi drivers from Fez, Morocco were enrolled from January to May 2013.We conducted this study to estimate the prevalence of fatigue among taxi drivers and to assess the determinant of fatigue among taxi drivers.

In this study we found that low income, cardiovascular disorders, tobacco use, problems with colleagues, regularity of sleep and sleeping at the wheel. In our study we found various possible causes of fatigue among taxi drivers in Morocco. The level of 
Table 3 Pichot scale and associated factors (Multiple Linear Regression).

\begin{tabular}{llll}
\hline & $\mathrm{B}$ & Adjusted IC (95\%) & $\mathrm{p}$ \\
\hline $\begin{array}{l}\text { Income } \\
\text { Mew income }\end{array}$ & 1 & - & \\
$\begin{array}{l}\text { Medium or high income } \\
\text { Cardiovascular disorders }\end{array}$ & -0.128 & {$[-0.030 ; 0.000]$} & 0.017 \\
Yes & 0.223 & {$[2.602 ; 7.365]$} & 0.000 \\
No & 1 & - & \\
Tobacco use & 1 & - & 0.010 \\
Yes & -0.141 & {$[-3.142 ;-0.439]$} & \\
No & 1 & - & 0.002 \\
Problems with colleagues & -0.165 & {$[-4.869 ;-1.066]$} & \\
Yes & 1 & - & 0.013 \\
No & 0.135 & {$[0.468 ; 3.908]$} & \\
Regularity of sleep & 1 & & 0.000 \\
Yes & -0.243 & {$[-5.837 ;-2.248]$} & \\
No & & & \\
Sleeping at the wheel & Yes & & \\
No & & & \\
\hline
\end{tabular}

income was associated with fatigue among our study population. We can easily explain this by the fact that many drivers have low income in Morocco and when they try to ameliorate their revenues they have to work for many continuous hours during the day and sometimes the night without having enough rest. Actually many studies found that the occupational stress can be related to some unfavorable job outlook, low income, lack of prestige, and underprivileged position [14]. According to many articles, Irregular sleeping habits and excessive stress are risk factors for fatigue (15). In our study, we searched many stress causes (as we have questions about the participant problems with clients, colleagues or authority) and we found that the problems with colleagues were associated with excess of fatigue. It's well known that stress can influence a person regularity of sleepiness and hence with a work which required many hours of concentration it can generate signs of fatigue. Many professional drivers have been sleeping at the wheel, but there is no significant increase in road accident related to fatigue.

This study revealed that some diseases like cardiovascular diseases can be associated to excess of tiredness among professional drivers. This is online with many reports which showed that professional drivers have a higher chance of developing cardiovascular diseases because of the greater psychiatric pressure in their working situations or their high workloads that include driving in heavy traffic, obesity, low physical activity, high demand and low decision latitude [16-18].

It's well-known that fatigue and the quality of sleep can affect the driver skills [19]. But the direct association between both fatigue and sleep disorder on one hand and the professional drivers' performance on the second hand is a topic under research [20-24]. Sleep deprivation can cause the vigilance and concentration decrease [25]. Pichot questionnaire is one of the most widely used questionnaires to assess fatigue among people. In this study we used this questionnaire to assess fatigue among healthy people (professional drivers) which can influence negatively their ability and can cause road accidents.

As limits of this study, we can imagine thatthe relationship between fatigue and car accidents can biased by many confounders, however in this survey we tried to cover most of the suspected factors and neutralize their effect by multivariate analysis. In Morocco many women drive their cars but no women are working as professional taxi drivers and hence in this study all participants were men and inter-gender comparisons were not possible. And the other limits of our study are the classic limits of 
cross-sectional studies like the difficulty to make causal inference. However, it was a pioneer study this is why we conducted a Cross-sectional study in order to give us a quick picture of the situation. These first results have to be confirmed by other epidemiological studies.

In the future, we recommend that many prevention programs should be developed including educational sessions for professional drivers with awareness sessions about using the rest areas. In addition, legislative or regulatory initiatives should also be discussed [26]. This study opened the door to many discussions about the professional drivers' conditions of work and we can argue that we should ameliorate the professional drivers conditions of work in Morocco.

\section{Ethical Aspect}

This study has been presented to the local ethics committee of University Hospital Hassan II of Fez who agreed it. This survey was classified as minimal risk research.

An oral informed consent has been taken from all participants after full explanations of the study and the participants' rights and before collecting any data. Questionnaires have been assigned a unique number and at any time during the analysis of the study no person had been cited in order to respect the confidentiality and the privacy of all participants.

\section{Authors Contribution}

NadiaBENAICHA: Conducting the survey, Statistical Analysis, Writing the article

Abdessalam DIARA: Statistical analysis

OussmaneSY: Statistical analysis

OussmaneDésiré DIAKITE: Statistical analysis

Adil NAJDI: Conducting and supervising the survey, reviewing the article

Mohamed Amine BERRAHO: Writing the protocol, writing the questionnaire, reviewing the Consent Form, reviewing the article

\section{Acknowledgments}

We thank the participants who accepted to answer the questions.

\section{Competing Interest}

The authors declare they have no competing interest.

\section{Funding}

The authors didn't have any funding for this survey.

\section{Availability of Data and Materials}

Data will not be shared: Having collected the data, our team wants to be the one to analyze it and to benefit from it. Other article about some other aspects of this study will be published. Once, all papers published, we can share our database.

\section{References}

[1] EbrahimRazmpa, KhosroSadeghNiat, and BabakSaedi Urban Bus Drivers' Sleep Problems and Crash Accidents Indian J Otolaryngol Head Neck Surg. 2011 Jul; 63(3): 269-273. Published online 2011 Apr 30. doi: 10.1007/s12070-011-0235-5[PubMed]

[2] Nathaniel S, Marshall WB, Philippa H. Gander: abnormal sleep duration and motor vehicle crash risk. J Sleep Res. 2004;13:177-178. doi: 10.1111/j.1365-2869.2004.00402.x. [PubMed] [Cross Ref]

[3] Kevin M. Sleep deprivation: a clinical perspective. Sleep Biol Rhythms. 2007;5:2-14. doi: 10.1111/j.1479-8425.2006.00251.x. [Cross Ref]

[4] D'Orazio T, Leo M, Guaragnella C, Distante A. A visual approach for driver inattention detection. Pattern Recogn. 2007;40:2341-2355. doi: 10.1016/j.patcog.2007.01.018. [Cross Ref]

[5] Kryger M, George C. Sleepy older drivers. CMAJ. 2007;177(4):376-377. [PMC free article] [PubMed]

[6] Chouvarda I, Papadelis C, Kourtidou-Papadeli C, Bamidis PD, Koufogiannis D, Bekiaris E, Maglaveras N. Non-linear analysis for the sleepy drivers' problem. Stud Health Technol Inform. 2007;12:1294-1298. [PubMed]

[7] Philip P. Sleepiness of occupational drivers. Ind Health. 2005 Jan; 43(1):30-3. [PubMed]

[8] Mitler MM, Miller JC, Lipsitz JJ, Walsh JK, Wylie CD (1997). The sleep of long-haul truck drivers. N Engl J Med $337,755-61$. 
[9] Kevin M. Sleep deprivation: a clinical perspective. Sleep Biol Rhythms. 2007;5:2-14. doi: 10.1111/j.1479-8425.2006.00251.x. [Cross Ref]

[10] Workplace safety and health guidelines - fatigue management. Ministry of Manpower and WSH Council. [Accessed January 30 2013];2010 Available at https: //www.wshc.sg/files/wshc/upload/cms/file/2014/Fatigue_ Management.pdf .

[11] http://www.sommeil-mg.net/spip/Pichot-s-fatigue-scale.

[12] Connor J, Norton R, Ameratunga S, et al. Driver sleepiness and risk of serious injury to car occupants: population based case control study. BMJ. 2002;324:1125. [PMC free article][PubMed]

[13] Murray C, Lopez A, editors. The global burden of disease: a comprehensive assessment of mortality and disabilityfrom diseases, injuries and risk factors in 1990 and projected to 2020. Global Burden of disease and Injury Series, 1st ed. Cambridge: Harvard University Press; 1996

[14] Yun Kyung Kim, and Nam Hyun Cha. Correlations among occupational stress, fatigue, and depression in call center employees in Seoul. nJPhysTher Sci. 2015 Oct; 27(10): 3191-3194. Published online 2015 Oct 30. doi: $10.1589 /$ jpts.27.3191

[15] Schwartz CE, Coulthard-Morris L, Zeng Q.: Psychosocial correlates of fatigue in multiple sclerosis. Arch Phys Med Rehabil, 1996, 77: 165-170. [PubMed]

[16] Hedberg GE, Jacobsson KA, Janlert U, Langendoen S. Risk indicators of ischemic heart disease among male professional drivers in Sweden. Scand J Work Environ Health. 1993. pp. 326-333. [PubMed]

[17] Hartvig P, Midttun O. Coronary heart disease risk factors in bus and truck drivers. Int Arch Occup Environ Health. 1983;25:353-360. doi: 10.1007/BF02226900. [PubMed][Cross Ref]
[18] Netterstrøm B, Juel K. Impact of work-related and psychosocial factors on the development of ischemic heart disease among urban bus drivers in Denmark. Scand J Work Environ Health. 1988. pp. 231-238. [PubMed]

[19] Acheson A, Richards JB, Wit H. Effects of sleep deprivation on impulsive behaviors in men and women. PhysiolBehav. 2007;91:579-587. doi: 10.1016/j.physbeh.2007.03.020.

[20] Benbadis SR, Perry MC, Sundstad LS, Wolgamuth BR. Prevalence of daytime sleepiness in a population of drivers. Neurology. 1999;52(1):209-210. [PubMed]

[21] Pandi-Perumal SR, Verster JC, Kayumov L, Lowe AD, Santana MG, Pires ML, Tufik S, Mello MT. Sleep disorders, sleepiness and traffic safety: a public health menace. Braz J Med Biol Res. 2006;39(7):863-871. doi: 10.1590/S0100-879X2006000700003. [PubMed] [Cross Ref]

[22] Carter T, Major H, Wetherall G, Nicholson A. Excessive daytime sleepiness and driving: regulations for road safety. Clin Med. 2004;4:454-456. [PubMed]

[23] Lucidi F, Russo PM, Mallia L, Devoto A, Lauriola M, Violani C. Sleep-related car crashes: risk perception and decision-making processes in young drivers. Accid Anal Prev. 2006;38:302-309. doi: 10.1016/j.aap.2005.09.013. [PubMed] [Cross Ref]

[24] Léger D, Guilleminault C, Bader G, Lévy E, Paillard M. Medical and socio-professional impact of insomnia. Sleep. 2002;25:625-629. [PubMed]

[25] Taillard J, Philip P, Chastang JF, Diefenbach K, Bioulac B. Is self-reported morbidity related to the circadian clock. $\mathrm{J}$ Biol Rhythms. 2001;16:183-190. doi: 10.1177/074873001129001764.

[26] Rosen IM. Driving while sleepy should be a criminal offense. J Clin Sleep Med 2005;1: 337-4. [PubMed 